\title{
Least Square Linearization of a Nonlinear System with Excitation-Application to a Nonlinear Junction Type Josephson
}

\author{
M.F. Bendahmane ${ }^{1}$, T. Benouaz ${ }^{2}$ and N. Ghouali ${ }^{2}$
}

\begin{abstract}
The aim of this work is to apply the 0ptimal Derivative procedure to a physical nonlinear system with excitation. We present a study of a nonlinear electronic circuit with excitation and the nonlinearity is the Josephson junction.
\end{abstract}

Index Terms-Nonlinear system - ordinary differential equation - optimal Derivative - Josephson junction.

\section{INTRODUCTION}

A class of ordinary differential equations is the nonlinear characteristic behaviour of electronic circuits. Among the infinity of possible temporal dependencies, the circuits in free regime and circuits with sinusoidal excitation play an important role.

These systems are primarily used free system to model the behaviour of circuits in the absence of signals. This steady state of the circuit before it is excited by a signal of finite duration and the return to steady state after injection thereof.

Systems with sinusoidal excitation modeless especially circuits of electric power distribution and transmission channels of modulated signals. One of the most important features of the behaviour of a circuit is his or her system solutions. The solution scheme is mainly dictated by the asymptotic behaviour of sources that depend on time.

We are interested in this study for physical systems modelling of electronic circuits which are excitedly usually described by systems of ordinary differential equations, nonlinear form

$$
\left\{\begin{array}{l}
\frac{d x}{d t}=F(x(t), u(t)) \\
x(0)=x_{0}
\end{array}\right.
$$

We apply the optimal method of derivation introduced by Arino-Benouaz [12, 13, 16, 18, 15], and a comparison is made from the square error between the nonlinear system and linear optimal obtained [20].

This work was supported by the CNEPRU- MESRS Research program. MESRS/2010.

T. Benouaz i is with the Laboratoire d'Automatique, B p 119 , Abu Bakr Belkaid University, Tlemcen, 13000, Algeria .(e-mail: T_benouaz@ mail-univ-tlemcen.dz).

Dr.M. F Bendahmane, was in with the Telecommunication laboratory, Department of physics, University of Tlemcen, B.p. 119, Tlemcen R.p

N. Ghouali,is was Laboratoire d'Automatique, B p 119 , Abu Bakr Belkaid University, Tlemcen, 13000, Algeria.

${ }^{1}$ Laboratoire de Télécommunications

${ }^{2}$ Laboratoire d'Automatique

\section{PRoblem}

Consider the system of ordinary differential equations non-linear form

$$
\left\{\begin{array}{l}
\frac{d x}{d t}=F(x(t), u(t)) \\
x(0)=x_{0}
\end{array}\right.
$$

Where

$$
\begin{aligned}
& x=\left(x_{1}, \cdots, x_{n}\right) \text { is the unknown function } \\
& u=\left(u_{1}, \cdots, u_{n}\right) \text { represents the excitement that can be } \\
& \text { constant (continuous), or variable with time (sinusoidal } \\
& \text { source) } \\
& f=\left(f_{1}, \cdots, f_{n}\right) \text { is a function defined in some open, }
\end{aligned}
$$
with values in $I R^{n}$.

In the absence of any stimulation $(u(t)=0)$, the system is at equilibrium.

We have the following assumptions:

H1) $F(0,0)=0$

H2) $F$ is, Lipchitz continuous of Lipchitz constant $\gamma$.

H3) The spectrum is contained in the set $\{z: \operatorname{Re} z<0\}$ for everyone $x \neq 0$ in a neighbourhood of 0 , which $D F(x)$ exists.

The problem that arises can be translated as follows:

Find a linear ordinary differential equation of the form

$$
\left\{\begin{array}{l}
\frac{d x}{d t}=\widetilde{A} x+\widetilde{B} u \\
x(0)=x_{0}
\end{array}\right.
$$

Approaching the nonlinear equation (1) with the same initial conditions such that the functional

$$
G(A, B)=\int_{0}^{+\infty}\|F(x(t), u(t))-A x(t)-B u(t)\|^{2} d t
$$

is minimal. $F(x(t), u(t))$ being as above, and $(\widetilde{A}, \widetilde{B}) \in M_{n}\left(I R^{n}\right)$ to determine.

The problem thus posed is type optimization in the least-squares sense. The aim is try to replace the original nonlinear equation by a linear equation. That means try to make an approximation of the solution of system (1) by that of (2). Minimizing the functional with respect to $A$ and $B$ is 
compared to solutions from the initial point and tend to the solution plan when $t \rightarrow+\infty$.

\section{FORMALISM}

Minimizing the functional is obtained by differentiating with respect to $A$ and $B$. This minimization allows us to calculate the optimal matrices $\widetilde{A}$ et $\widetilde{B}$ such as

$$
\left\{\begin{array}{l}
D G(A) \alpha=2 \int_{0}^{+\infty}\langle A x(t)+B u(t)-F(x(t),(u(t))), \alpha x(t)\rangle d t \\
D G(B) \beta=2 \int_{0}^{+\infty}\langle A x(t)+B u(t)-F(x(t),(u(t))), \beta u(t)\rangle d t
\end{array}\right.
$$

for all matrices $\alpha$ and $\beta$, in particular matrices such that

$$
\left\{\begin{array}{l}
\alpha_{l, m}=1 ; \alpha_{i j}=0 \\
\beta_{l, m}=1 ; \beta_{i j}=0
\end{array} \text { If }(i, j) \neq(l, m)\right.
$$

After calculations [16.18], and with

$$
\left\{\begin{array}{l}
\Gamma_{A}(x)=\int_{0}^{+\infty}[x(t)][x(t)]^{T} d t \\
\Gamma_{B}(u)=\int_{0}^{+\infty}[u(t)][u(t)]^{T} d t \\
\phi_{A}(u, x)=\int_{0}^{+\infty}[u(t)][x(t)]^{T} d t \\
\phi_{B}(x, u)=\int_{0}^{+\infty}[x(t)][u(t)]^{T} d t \\
\Psi_{A}(x, u)=\int_{0}^{+\infty}[F(x(t), u(t))][x(t)]^{T} d t \\
\Psi_{B}(x, u)=\int_{0}^{+\infty}[F(x(t), u(t))][u(t)]^{T} d t
\end{array}\right.
$$

we obtain

$$
\left\{\begin{array}{l}
A \Gamma_{A}(x)+B \Phi_{A}(u, x)=\Psi_{A}(x, u) \\
A \Phi_{B}(u, x)+B \Gamma_{B}(u)=\Psi_{B}(x, u)
\end{array}\right.
$$

This allows us to have the matrices $A$ and $B$ as

$\left\{\begin{array}{l}A=\left[\Psi_{A}(x, u)-(B) \phi_{A}(u, x)\right]\left[\Gamma_{A}(x)\right]^{-1} \\ B=\left[\Psi_{B}(x, u)-(A) \phi_{B}(x, u)\right]\left[\Gamma_{B}(u)\right]^{-1}\end{array}\right.$

The matrices $A$ and $B$ are defined and unique if that the functions $\Gamma_{A}(x)$ and $\Gamma_{B}(x)$ are invertible.

\section{PROCEDURE}

\section{A. Schematic procedure of the optimal derivative}

The diagram of the procedure derived optimal is given in the following diagram

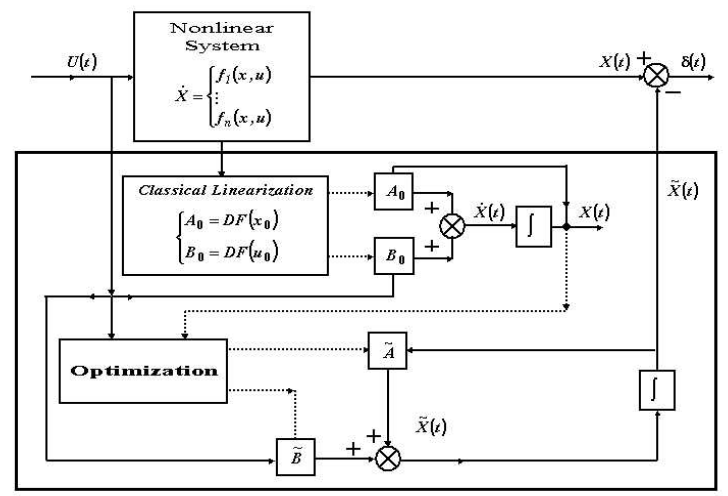

Fig. 1 Schematic procedure of the optimal derivative

\section{B. Computational Procedure.}

We will use the preceding calculation iteratively. Assume that successive matrices $A_{j}$ and $B_{j}$ are stable, i.e. its spectrum is contained within $\{z: \operatorname{Re} z<0\}$. The initial matrices $A_{0}$ and $B_{0}$ are respectively the Jacobian matrices of $F$ at $x_{0}$ where $D F(x)$ exists, and $u_{0}$ where $D F(u)$ exists. $x_{0}$ is the initial condition and $u_{0}$ represents the excitation at $t=0$. Consider the system (1)

$$
\left\{\begin{array}{l}
\frac{d x}{d t}=F(x(t), u(t)) \\
x(0)=x_{0}
\end{array}\right.
$$

First step

$$
\text { Compute }\left\{\begin{array}{l}
A_{0}=D F\left(x_{0}\right) \\
B_{0}=D F\left(u_{0}\right)
\end{array}\right.
$$

\section{Second step}

Compute $A_{1}$ and $B_{1}$ from the solution of the equation

$$
\left\{\begin{array}{l}
\frac{d x}{d t}=A_{0} y(t)+B_{0} v(t) \\
y(0)=x_{0}
\end{array}\right.
$$

which reads as follows

$$
y(t)=e^{A_{0} t} x_{0}+\int_{0}^{t} e^{(t-s) A_{0}} B_{0} v(s) d s
$$

minimizing the functional

$$
G(A, B)=\int_{0}^{+\infty}\|F(y(t), v(t))-A y(t)-B v(t)\|^{2} d t \text {. }
$$

$A_{1}$ and $B_{1}$ are determined uniquely by the system of equations (8), where $x$ is replaced by $y$ and $v(t)$ is the excitation at time $t$.

This shows that apart from the initial matrices, the matrices determined by the procedure are not the Jacobian matrix of $F$ at a given point. Subsequently, it is necessary that the conditions for the start of the study are met at every step. If we assume that this is true then the procedure works as follows

\section{Third step}

Assuming that $A_{1}, \ldots, A_{j-1}$ and $B_{1}, \ldots, B_{j-1}$ have been computed, to compute $A_{j}$ from $A_{j-1}$ and $B_{j}$ from de $B_{j-1}$, we must first solve the following system 


$$
\left\{\begin{array}{l}
\frac{d x}{d t}=\left[A_{j-1}\right] y(t)+\left[B_{j-1}\right] v(t) \\
y(0)=x_{0}
\end{array}\right.
$$

Let $y_{j}$ the solution of equation (12) as

$$
y_{j}(t)=e^{A_{(j-1)} t} x_{0}+\int_{0}^{t} e^{(t-s) A_{(j-1)}} B_{(j-1)} v_{j}(s) d s
$$

and $v_{j}$ is the excitation at time $t$.

Minimizing the functional

$$
G_{j}(A, B)=\int_{0}^{+\infty}\left\|F\left(y_{j}(t), v_{j}(t)\right)-A y_{j}(t)-B v_{j}(t)\right\|^{2} d t
$$

leads to

$$
\left\{\begin{array}{l}
{\left[A_{j}\right]=\left[\int_{0}^{+\infty}\left[F\left(y_{j}(t), v_{j}(t)\right)\right]\left[y_{j}(t)\right]^{T} d t-\left(B_{j}\right)\left(\phi\left(v_{j}, y_{j}\right)\right)\right]\left[\Gamma\left(y_{j}\right)\right]-1} \\
{\left[B_{j}\right]=\left[\int_{0}^{+\infty}\left[F\left(y_{j}(t), v_{j}(t)\right)\right]\left[v_{j}(t)\right]^{T} d t-\left(A_{j}\right)\left(\phi\left(y_{j}, v_{j}\right)\right)\right]\left[\Gamma\left(v_{j}\right)\right]-1}
\end{array}\right.
$$

If the suites $\left(A_{j}, B_{j}\right)$ converges, then $(\widetilde{A}, \widetilde{B})$ represent the derivation of the $F(x(t), u(t))$ in the point $\left(x_{0}, u\right)$

\section{APPLICATION}

We present in this section an application relating to the procedure of the optimal derivative of a system of nonlinear ordinary differential equations with excitation, by applying the procedure developed above. The nonlinearity is concerning the non-linear Josephson junction.

The majority of the research work of the Quantum Nanoelectronics Group is based on Josephson junctions. The Josephson junction was predicted by Brian Josephson in 1962, for which he won the Nobel Prize for Physics in 1973. In its most general form it consists of two superconductors separated by a thin layer of something which is not superconducting. The interference between the wave functions of the superconducting condensate on either side of this non-superconducting barrier leads to a variety of interesting quantum phenomena, many of which have been (or can be) exploited in applications

This will allow us to illustrate by comparing curves or by calculating a quadratic error, the results obtained. The calculation of the quadratic error is using the following relation

$$
E r=\sum_{n=1}^{n}\|x(t)-\widetilde{y}(t)\|
$$

$x(t)$ represents the solution of nonlinear system,

$\tilde{y}(t)$ represents the solution of the system obtained from the optimal Derivative. For this application, we consider a physical system modelling a simple electronic circuit (Figure.2.)

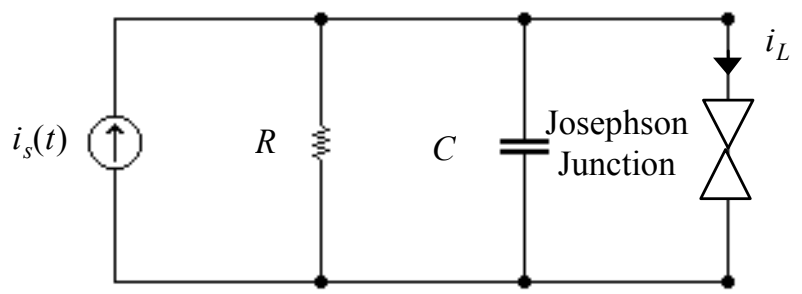

Fig.2. Electronic circuit with a nonlinear junction

This is a circuit with two state variables (voltage drop $v_{C}$ across the terminals of the capacity and $\phi_{L}$ flow of the Josephson junction), whose nonlinearity is the presence of the non-linear Josephson junction [10].

$$
i=I_{0} \sin (K \phi)
$$

The laws of Kirchhoff relating to the nodes and the meshes of the circuit give us the following equations of state

$$
\left\{\begin{array}{l}
\frac{d v_{C}}{d t}=\frac{1}{C}[-R v_{C}-\underbrace{I_{0} \sin \left(K \phi_{L}\right)}_{i_{L}}+i_{s}(t)] \\
\frac{d \phi_{L}}{d t}=v_{C}
\end{array}\right.
$$

We consider that the capacity $C$ was initially charged by a voltage $v_{C_{0}}$, so the initial conditions of the nonlinear system are

$$
\left(v_{C_{0}}, \phi_{L_{0}}\right)=(10,0)
$$

The parameters chosen in the circuit are

$$
\begin{aligned}
& C=1 F \quad R=0.25 \Omega \\
& I_{0}=1 \quad K=1 \\
& i_{s}(t)=\cos (100 \pi t) A
\end{aligned}
$$

We set

$$
\left\{\begin{array}{l}
x=v_{C} \\
y=\phi_{L}
\end{array}\right.
$$

then the system (18) becomes, taking into account the values of circuit components, as

$$
\left\{\begin{array}{l}
\frac{d x}{d t}=-0.25 x-\sin (y)+u(t) \\
\frac{d y}{d t}=x
\end{array}\left(x_{0}, y_{0}\right)=(10,0)\right.
$$

with

$$
u(t)=\frac{1}{C} i_{s}(t) H(t)=[\cos (100 \pi t)] H(t)
$$

$H(t)$ being the Heaviside function.

By applying the procedure developed above and after six iterations ( $\varepsilon=10^{-6}$ ), the optimal linear system can be written

$\left[\begin{array}{l}\dot{x} \\ \dot{y}\end{array}\right]=\left[\begin{array}{cc}-0.26157 & -0.51985 \\ 1 & 0\end{array}\right] \cdot\left[\begin{array}{l}x \\ y\end{array}\right]+\left[\begin{array}{ll}1 & 0 \\ 0 & 0\end{array}\right] u(t)$ 
Figures (3) and (4) represent the respective components of the solution of systems (22) and (24) with respect to time. Figure (5) represents the quadratic error with respect to time.

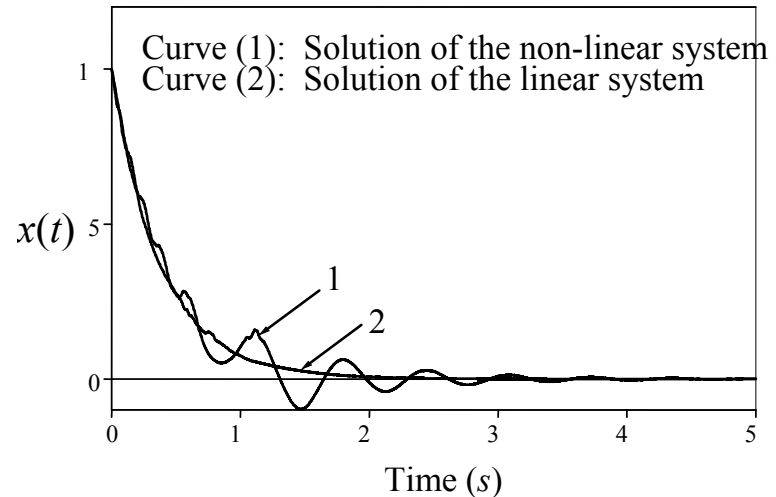

Fig.3. Variation of the solution $\mathrm{x}(\mathrm{t})$ with respect to time for the initial conditions $(\mathrm{x} 0, \mathrm{y} 0)=(10,0)$

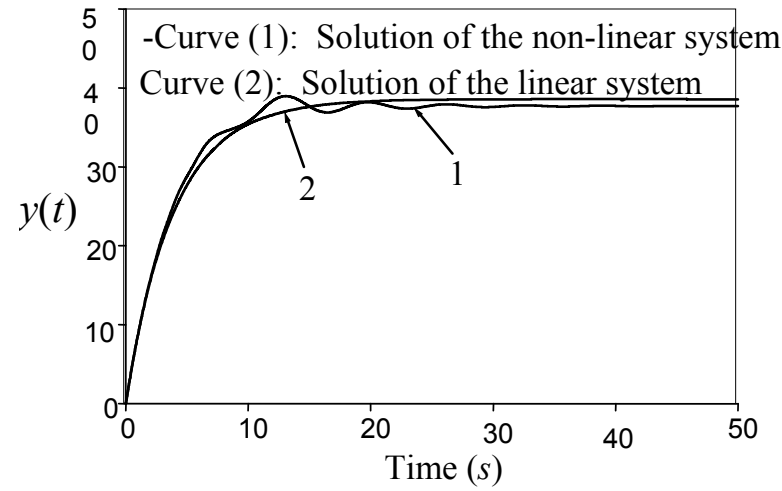

Fig.4. Variation of the solution $y(t)$ with respect to time for the initial conditions $(\mathrm{x} 0, \mathrm{y} 0)=(10,0)$

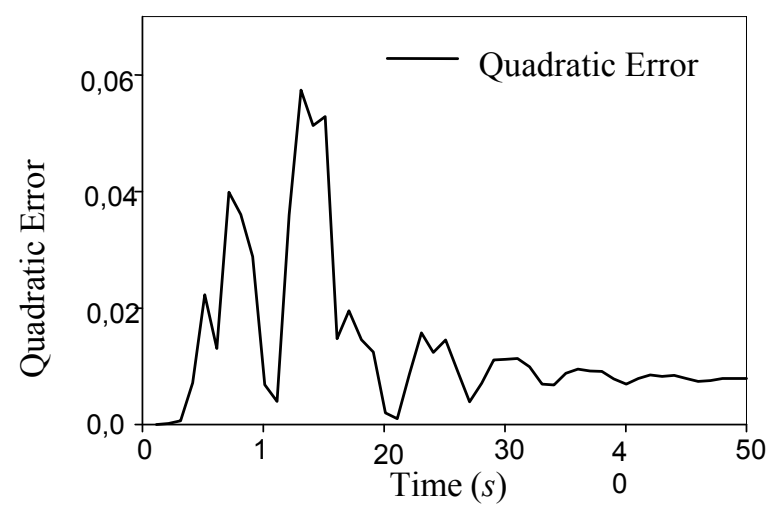

Fig.5. The quadratic error as a function of time between the nonlinear system (22) and the optimal linear system (24)

Note that the solution given by the optimal derivative is of the same order of magnitude as that given by the nonlinear equation. It makes it possible to bring the system after excitation, of its position of balance towards its point of operation (or solution plan). This is attested by the study of the quadratic error which reaches its maximum at time $t=13$ $s, 5.73 \%$ and thus remains small.

This error becomes too small when $t \geq 30 \mathrm{~s}$ i.e. when the solutions reach the point of operation of the electronic circuit. At this level the solution of the nonlinear system is almost confused with that of the optimal linear system.

\section{CONCLUSION}

The exposed example shows that the approximation obtained by the optimal derivation gives satisfactory results compared to the exact results while respecting the dynamics of the initial problem.

We note that although the solutions obtained converge to the plan solution when $t \rightarrow+\infty$.

\section{REFERENCES}

[1] A. Fossard, Commande des Systèmes Multidimensionnels , Dunod, 1972.

[2] B. Vujanovic, B. Baclic, Application of Gauss Principle of Least Constraint to the Non-Linear Heat Transfer Problem, Int. Heat. Mass Transfer, Vol.13, pp.721-730. Pergamon Press, Oxford, 1975.

[3] A. Jordan, M. Benmouna, A. Bensenane, A. Borucki, Optimal Linearization Method Applied to the Resolution of State Equation, RAIRO-APII, 21, pp.175-185, 1987.

[4] A. Jordan, M. Benmouna, A. Bensenane, A. Borucki, Optimal Linearization of Non-Linear State Equation, RAIRO-APII, 21, pp.263-271, 1987.

[5] J. P. Demailly, Analyse Numérique et Equations Différentielles, Presses universitaires de Grenoble, 1991.

[6] H. Reinhart, Equations Différentielles, Fondements et Applications, Gauthier-Villars, 1982.

[7] M. Siboni, J. CI. Mardon, Approximations et Equations Différentielles. Analyse Numérique II, Hermann, Editeurs des Sciences et des Arts, 1988.

[8] E.A. Coddington, N. Levinson, Theory of Ordinary Differential Equations, Mc Graw-Hill, 1955.

[9] W. Ralston, Mathematical Methods for Digital Computers, Wiley, New York, pp.110-120, 1960.

[10] L.O. Chua, Ch.A. Desoer, E.S. Kuh, Linear and Non-linear Circuits, McGraw-Hill Book Company, 1987.

[11] M. Hasler, J. Neirync, Circuits Non Linéaires, Presses Polytechniques Romandes, 1985.

[12] T. Benouaz, O. Arino, Existence, Unicité et Convergence de l'Approximation au Sens des Moindres Carrés d'une Equation Différentielle Ordinaire Non Linéaire, 94/14 Université de Pau, CNRS URA 1204, 1994.

[13] T. Benouaz, O. Arino, Relation Entre l'Approximation Optimale et la Stabilité Asymptotique, Publications de l'U.A, CNRS 1204, N95/10, 1995.

[14] T. Benouaz, Optimal Derivative of a Non-Linear Ordinary Differential Equation, Equadiff 99, International Conference on Differential equations, Berlin (Germany) 1-7 August, Vol.2, pp.1404-1407, World scientific Publishing Co. Pte. Ltd, 2000.

[15] T. Benouaz, O. Arino, Optimal Approximation of the Initial Value Problem, Computers Math. Applic., Vol.36, N¹, pp.21-32, 1998.

[16] T. Benouaz, O. Arino, Determination of the Stability of a Non-linear Ordinary Differential Equation by Least Square Approximation. Computational Procedure, Appl. Math. and Comp sci., Nº, Vol.5, pp.33-48, 1995.

[17] T. Benouaz, Lyaponov Function Generated by Least Square Approximation, Deuxième Conférence Maghrébine sur l'Automatique, Tlemcen (Algeria) 3-5 December, Vol.1 pp.73-75, 1996.

[18] T. Benouaz, O. Arino, Least Square Approximation of a Non-Linear Ordinary Differential Equation, Computers Math. Applic., Vol.31, $\mathrm{N}^{\circ} 8$, pp.69-84, 1996.

[19] M.F. Bendahmane, Contribution à l'Etude des Systèmes Non Linéaires avec Excitation, Thèse de Magister, Tlemcen (Algeria), 2000.

[20] T. Benouaz, M.F. Bendahmane, Least-Square Approximation of a Nonlinear O.D.E. with Excitation, Computers \& Mathematics with Applications, Vol. 47, pp. 473-489, 2004

Professor T Benouaz works as a Teaching, Department of Physics, Tlemcen University. Director of Post graduation of Electronic Physical Modeling in the Same university. His current research interest includes the computational physics, modeling and simulation of the nonlinear systems, applied mathematics. Director several research projects and has several publications in this field. The last publications: "Numerical simulation of nonlinear 
pulses propagation in a nonlinear optical directional coupler", International Journal of Physical Sciences Vol. 4 (9), pp. 505-513, September, 2009.ISSN 1992 - 1950 (C) 2009 Academic Journals. "On the Relationship between the Optimal Derivative and Asymptotic Stability ", African Diaspora Journal of Mathematics. Special Issue in Memory of Prof. Ibni Oumar Mahamat Saleh. Volume 8, Number 2, pp. 148-162 (2009) "Simulation and Experimental Studies of an Internal Thermal Insulation of two Pieces of Rooms Located in Ghardaia (Algeria)", International Journal of Advanced Computer Engineering , January- June 2009, Volume 2, issue 1. "Prediction model for the diffusion lengh in silicon-based solar cells", Journal of Semiconductors , Vol.30, $\mathrm{N}^{\circ} .5$, pp. 40001-1-40001-4, May 2009.

Professor Nouredinne Ghouali, is was the head of Laboratory of Automatique (2000-2009). The interesting work is Dynamical Systems, Power systems . His current research interest includes the computational Physics, modeling and simulation of the nonlinear systems, applied mathematics. Director of several research projects and has several publications in this field.

Dr.M. F Bendahmane, was in with the Telecommunication laboratory, Department of physics, University of Tlemcen, B.p. 119, Tlemcen R.p . 